\title{
Influence of viscosity on the growth of human gut microbiota
}

2 Alba Tamargo ${ }^{\mathrm{a}}$, Carolina Cueva ${ }^{\mathrm{a}}$, M. Dolores Alvarez, Beatriz Herranz ${ }^{\mathrm{b}}$, Begoña

3 Bartoloméa, M.Victoria Moreno-Arribas ${ }^{\mathrm{a}}$, Laura Laguna*a

4

5

6 anstitute of Food Science Research (CIAL), CSIC-UAM

7 C/ Nicolás Cabrera 9, 28049, Madrid, Spain

8 bInstitute of Food Science, Technology and Nutrition (ICTAN), CSIC

9 C/ José Antonio Novais, 10, 28040 Madrid, Spain

10

11

12

$21{ }^{*}$ Corresponding author

22 Tel: (00)34 910017900- 822 


\section{Abstract}

Numerous studies support the beneficial effects of dietary fibre. It is well known that fibre increases viscosity at intestinal level. Therefore, the effects of fibre on gut microbiota could be due not only by its intestinal bacteria fermentation but also to the increase in viscosity by itself. The aim of this study was to evaluate the effect of viscosity on the growth of gut microbiota at physiological conditions. For this purpose, four compartments from a gastrointestinal simulator (simgi ${ }^{\circledR}$ ) were filled with Gut Nutrient Medium (GNM) plus different agar concentrations (0, 0.30, 0.45 and $0.60 \%$ ), inoculated with faecal microbiota, and incubated $48 \mathrm{~h}$ under anaerobic conditions. Samples were collected at three time points $(0,24 \mathrm{~h}$ and $48 \mathrm{~h})$ for representative intestinal bacterial enumeration and rheological characterization. Incubation of GNM gels with faecal microbiota changed the medium viscosity over time, even with constant conditions (temperature and $\mathrm{pH}$ ). In such way that, in absence of agar (low viscosity), viscosity slightly increased over time; however, in viscous mediums, viscosity decreased over time. In relation to the growth of gut microbiota, results showed that viscosity favoured the growth of total anaerobes and Clostridium spp.; in contrast, total number of aerobes and members of Enterococcus correlated negatively with viscosity increment. In conclusion, changes in intestinal viscosity seem to selectively modify microbiota composition. This is a pioneer work to understand the effect of food viscosity in the gastrointestinal system, showing that viscosity is an important factor itself to condition the growth of different bacteria’s groups.

Keywords: viscosity, faecal microbiota, bacteriological agar, gastrointestinal model, simgi ${ }^{\circledR}$

\section{Highlights}

- Viscosity changes by agar concentration influences the growth of gut microbiota

- Growth of total anaerobes are favoured by $\eta$ increment

- Medium viscosity changes over time depending on agar concentration 


\section{Introduction}

Dietary fibre refers to food components that are not digestible by gastrointestinal enzymes whereas functional fibre is referred to indigestible carbohydrates extracted or manufactured from plants (Gallagher, 2016). Both fibres (dietary and functional) have beneficial physiological effects, attributed to their degree of fermentation and viscosity (Slavin, 2005). The fermentation effect is related to the use of fibre as energy source by the microbiota, although effects vary depending on both the type of fibre and the individual's gut microbiota (Benítez-Páez et al., 2016). The increment of intestinal viscosity caused by fibre ingestion has been proven in in vitro studies (Dikeman, Murphy, \& Fahey, 2006) and in animals models (Danielson, Newman, Newman, \& Berardinelli, 1997; Ellis, Roberts, Low, \& Morgan, 1995). An increment of viscosity due to fibre is related to effects on serum cholesterol reduction (Trumbo, Schlicker, Yates, \& Poos, 2002), alteration in blood glucose, prolonged gastric emptying and slower transit time through the small intestine (Schneeman, 1987). However, remains unknown if viscosity, by itself, and no due to microbial fermentation has any effect on the bacterial growth.

In looking for an increment in the intestinal viscosity without fermentation effect for intestinal bacteria (Kim, Lee, Kim, \& Choi, 2012), agar could be a good choice. Agar is a mixture of marine polysaccharides: agarose (a neutral polymer) and agaropectin (a sulphated polysaccharide of low gel strength) (Marinho-Soriano \& Bourret, 2003). Agar gels tend to be rigid and brittle; however, if a shear stress is applied whilst gelation, a fluid gel will be formed (Bradbeer, Hancocks, Spyropoulos, \& Norton, 2015) and it will not form a rigid gel, being able to use agar with constant agitation (i.e. constant stirring for microbial growth).

Thus, the main objective of the current work was to determine the relationship between viscosity and the growth of human gut microbiota by the use of an in vitro gastrointestinal model system called simgi ${ }^{\circledR}$. Viscosities in gut nutrient medium (GNM) were adjusted with different percentages of agar in its fluid gel form $(0,0.30,0.45$ and $0.60 \%, w / v)$ and were inoculated with faecal slurry. Rheological properties and microbial counts were analyzed over a period of $48 \mathrm{~h}$.

\section{Materials and methods}

\subsection{Gut Nutrient Medium (GNM)}

To support the growth of the microbial ecology of the gastro-intestinal tract nutrients broths are needed, as the GNM used in this study. GNM composition was 
validated previously for a simulator of the human intestinal microbial ecosystem (SHIME) (Molly, Woestyne, Smet, \& Verstraete, 1994; Molly, Woestyne, \& Verstraete, 1993) and for simigi ${ }^{\circledR}$ (Cueva et al., 2015). The GNM contains glucose (Difco ${ }^{\mathrm{TM}}$, BD) (0.4 g/L), arabinogalactan (Sigma-Aldrich, Merk) (1 g/L), pectin from citrus peel (Sigma-Aldrich, Merk) (2 g/L), xylan from beechwood (Megazyme) (1 g/L), soluble starch (Difco $\left.{ }^{\mathrm{TM}}, \mathrm{BD}\right)(3 \mathrm{~g} / \mathrm{L})$, dextrose (Difco $\left.{ }^{\mathrm{TM}}, \mathrm{BD}\right)(0.4 \mathrm{~g} / \mathrm{L})$, yeast extract (Oxoid, ThermoFisher Scientific) (3 g/L), special peptone (Oxoid, ThermoFisher Scientific) (1 $\mathrm{g} / \mathrm{L}$ ), mucin from porcine stomach (Sigma-Aldrich, Merk) (4 g/L) and L-cysteine (Panreac AppliChem) (0.5 g/L). All compounds were dissolved in $1 \mathrm{~L}$ of distilled water and sterilized at $121{ }^{\circ} \mathrm{C}$ for $21 \mathrm{~min}$. The fermentable ingredients (or the energy source used by microorganism) were glucose, arabinogalactan, pectin, xylan, soluble starch, dextrose, yeast extract, peptone and mucin.

\subsection{Fluid gel production}

Preliminary experiments confirmed that agar was not degraded by human microbiota. Agar fluid gels were prepared by dispersing the different agar (Bacto ${ }^{\mathrm{TM}}$ Agar, BD) concentrations $(0,0.30,0.45$ and $0.60 \% \mathrm{w} / \mathrm{v})$ in $300 \mathrm{~mL}$ of GNM at room temperature in a magnetic stirrer $\left(25^{\circ} \mathrm{C}\right)$ in a $500 \mathrm{~mL}$ screwcap bottle. Lower concentrations of agar created no difference in viscosity, and higher concentrations were forming a solid gel. Before autoclaving at $121{ }^{\circ} \mathrm{C}$ during $21 \mathrm{~min}$, the GNM gel (medium enriched with agar) was adjust to final $\mathrm{pH}$ 6.3. After that, inside the autoclave, samples were lowered to 85 ${ }^{\circ} \mathrm{C}$ and incubated overnight in an orbital shaker (Minitron, Infors HT, Switzerland) at 150 rpm at $37^{\circ} \mathrm{C}$ (see Fig. 1).

\subsection{Faecal slurry}

The faecal slurry $(20 \%, w / v)$ to be inoculated into the simgi ${ }^{\circledR}$ compartments was prepared from fresh faecal sample of a healthy volunteer who had not received any antibiotic treatment in the prior six months of the experiment. Faecal samples were taken in anaerobic conditions. To dilute the faecal samples, a phosphate saline buffer $(0.1 \mathrm{M}, \mathrm{pH}$ 7) was used. The dilution was homogenized on a stomacher (Stomacher 400 Circulator, SEWARD, U.K.) at $230 \mathrm{rpm}$ during $3 \mathrm{~min}$.

\subsection{Simgi ${ }^{\circledR}$ experimental set up}

Simgi ${ }^{\circledR}$ is a computer-controlled dynamic SIMulator of the GastroIntestinal tract consisting of five successive reactors (Barroso et al., 2015; Cueva et al., 2015). 
However, in the present work, simgi ${ }^{\circledR}$ is used in a static form to control in vitro conditions, and four compartments worked as separate reactors with the same conditions ( $\mathrm{pH}$ and temperature) not connected among them.

GNM gels (agar-containing media) (300 mL) with different agar concentrations were transferred to the four intestinal simgi ${ }^{\circledR}$ compartments (used as independent reactors), then $20 \mathrm{~mL}$ of faecal slurry were added. For microbiological and rheological analysis, samples from the different simgi ${ }^{\circledR}$ compartments were taken at 3 different times: at $0 \mathrm{~h}$, $24 \mathrm{~h}$ and $48 \mathrm{~h}$. For better understanding, the experimental set-up is shown in Fig 1.

Same experimental conditions were kept for the four compartments during the whole experiments: $\mathrm{pH}$ was automatically regulated at $6.3 \pm 0.2 \mathrm{by} \mathrm{pH}$ controllers (Unitronic Vision $120^{\mathrm{TM}}$ ) and compartments were maintained at $37^{\circ} \mathrm{C}$ under anaerobic conditions by continuously flushing nitrogen.

\subsection{Viscosity measurements}

The rheological properties of the samples were measured in a Kinexus pro rheometer (Malvern Instruments Ltd., Worcestershire, UK), equipped with a $40 \mathrm{~mm}$ cone and plate and a gap of $0.150 \mathrm{~mm}$. Samples were placed on to the plates at $37{ }^{\circ} \mathrm{C}$ and a temperature cover was used to maintain the samples at the specified temperature. Temperature was controlled to within $0.1{ }^{\circ} \mathrm{C}$ by Peltier elements in the lower plates kept at $37^{\circ} \mathrm{C}$. To homogenize mechanical equilibrium before measurements a pre-shearing test of all samples was carried out during one min at $10 \mathrm{~s}^{-1}$ and at $37^{\circ} \mathrm{C}$. Then, flow curves were obtained as a function of shear rate ranging from $0.001-100 \mathrm{~s}^{-1}$. All samples were measured at least by triplicate.

For the statistical analysis of the flow curves, viscosities in two shear rates at physiological range ( 1 and $10 \mathrm{~s}^{-1}$ ) were taken (Hardacre, Lentle, Yap, \& Monro, 2016).

\subsection{Microbiological analysis}

Decimal dilutions of Simgi ${ }^{\circledR}$ samples in a physiological solution were plated on eight types of selective media as follows: Tryptic Soy Agar (TSA) (Becton and Dickinson and Company, BD) for total aerobes, Wilkins-Chalgren agar (BD) for total anaerobes, MacConkey agar (BD) for Enterobacteriaceae, Enterococcus agar (BD) for enterococci, MRS agar (CONDA-Pronadisa) for lactic acid bacteria, Tryptose Sulfite Cycloserine agar (TSC) (CONDA-Pronadisa) for Clostridium spp., Mannitol Salt agar (BD) for Staphylococcus aureus and LAMVAB for specific faecal Lactobacillus species (Hartemink, Domenech \& Rombouts, 1997). 
All plates were incubated at $37^{\circ} \mathrm{C}$ for $24-48 \mathrm{~h}$ in an anaerobic chamber (BACTRON Anaerobic/Environmental Chamber, SHELLAB, USA), except for Mannitol and TSA, which were incubated in aerobic conditions (Nüve Incubator EN 120, NÜVE, Turkey). Plate counting was done by triplicate. Results were expressed as colony-forming unit (CFU)/mL.

\subsection{Statistical analysis}

Analysis of variance (one-way ANOVA) was used to study the differences among agar concentrations and differences among incubation time within the same agar concentration. Least significant differences were calculated by Tukey’s test $(p<0.05)$. Two-way ANOVA was applied to study agar concentration and time main effects and interaction in the viscosity. Finally, to study the correlation between viscosity and bacteria growth, Pearson's correlation was done. Analyses were carried out using IBM SPSS Statistics for Windows, Version 22.0. Armonk, NY: IBM Corp.

\section{Results and discussion}

The flow curves of the GNM with different agar concentration (GNM gels without faecal slurry) are shown in Fig 2. As expected, GNM without agar was much less viscous than GNM gels with agar concentrations of $0.30,0.45$ and $0.60 \%$, being viscosity $(\eta)$ in all samples dependent on the shear rate from 0.001 to $10 \mathrm{~s}^{-1}$. After $10 \mathrm{~s}^{-}$ 1 , samples showed different shear dependence without agar arrived to an asymptote. However GNM gels with agar at the three different concentrations (0.30, 0.40 and 0.60 $\%$ of agar) had shear dependence at higher shear rates.

In table 1 it can be observed the GNM gels with faecal slurries at different times. In absence of agar (see Table 1 , agar concentration= $0 \% \mathrm{w} / \mathrm{v}$ ), viscosity slightly increased over time (from $\eta_{\mathrm{a}, 1}=0.005 \pm 0.001$ Pa.s to $0.009 \pm 0.001$ Pa.s). In opposition, in presence of agar (agar concentration $=0.30,0.45,0.60 \% \mathrm{w} / \mathrm{v}$ ), viscosity decreased over time (from $\eta_{\mathrm{a}, 1, \mathrm{t}=0}=0.421 \pm 0.056$ Pa.s to $\eta_{\mathrm{a}, 1, \mathrm{t}=48 \mathrm{~h}}=0.161 \pm 0.027$ Pa.s for $0.30 \%$ agar, from $\eta_{\mathrm{a}, 1, \mathrm{t}=0}=0.907 \pm 0.009$ Pa.s to $\eta_{\mathrm{a}, 1, \mathrm{t}=48 \mathrm{~h}}=0.430 \pm 0.069$ Pa.s for $0.45 \%$ agar, and from $\eta_{\mathrm{a}, 1, \mathrm{t}=0}=0.762 \pm 0.070$ Pa.s to $\eta_{\mathrm{a}, 1, \mathrm{t}=48 \mathrm{~h}}=0.548 \pm 0.114$ Pa.s for $0.60 \%$ agar).

The present results showed that mixtures of GNM gels with faecal slurry became more fluid with the passing of the days. Previous authors have mentioned that gel viscosity changes over time can be as consequence of $\mathrm{pH}$ or temperature changes (Holloway, Lowman, \& Palmese, 2013). Yet, as it was mentioned in section 2.4, pH and 
temperature were monitored and kept constant and in an air-tight container. At this point, it is essential to remind that bacteria were not able to decompose agar, and also, that same quantity of GNM fermentable nutrients were initially added to the four simgi ${ }^{\circledR}$ compartments (arabinogalactan, glucose, pectin, xylan, soluble starch, dextrose, peptone and mucin). However, due to the different viscosity created by the agar, availability of GNM nutrients, water and oxygen circulation flow in viscous media decreased, a fact that influences in vitro growth (Beruto, Beruto, \& Debergh, 1999). Then two connected effects might be happening. First, as the glucose is less available bacteria started to ferment the polysaccharides added to the GNM as arabinogalactan, pectin, soluble starch and mucin. These polysaccharides initially helped to the GNM gel structure, providing greater viscosity at the beginning and losing the structure when they are fermented. This fermentation caused short chain fatty acids (SCFA) (Macfarlane 2003) that decreased the $\mathrm{pH}$. As simgi ${ }^{\circledR}$ controls $\mathrm{pH}$ by adding $\mathrm{NaOH}$, a dilution of the GNM gels might happen. Although $\mathrm{NaOH}$ was added also to the GNM without agar $(0 \%$ of agar $\mathrm{w} / \mathrm{v})$, this was already fluid, so not changes in viscosity were registered.

The counting of bacteria at different agar concentrations over the time is shown in Fig. 3. As happened with the viscosity, not only the agar concentration, but also the time significantly influenced bacteria growth, although the pass of the time had higher influence $\left(\mathrm{F}_{\text {time }}>\mathrm{F}_{\text {agar }}\right.$ concentration, $\mathrm{P}_{\text {day,agar }}$ concentration $\left.<0.05\right)$. Additionally, Pearson' correlation between viscosity and bacteria population at both shear rates (1 and $\left.10 \mathrm{~s}^{-1}\right)$ were calculated (Fig. 3). Total aerobes (Fig. 3a), members of the Enterobacteriaceae family (Fig. 3c) and Enterococcus (Fig. 3d) showed lower growth than the rest of bacteria at any agar concentration and its growth was inverse to the viscosity increase; this tendency of growth was related with the lacking of oxygen that aerobes families needed. However, total anaerobes (Fig. 3b) and Clostridium spp. (Fig. 3f) grew significantly more with viscosity increment. The rest of the bacterial groups evaluated showed no significant changes in their growth with different viscosities. This viscosity dependence produced that obligate anaerobes grew significantly more as they undergo fermentation in the absence of oxygen

These results showed that gut bacteria growth displayed different behavior regarding viscosity depending on bacteria species. This fact is in accordance with preliminary studies where it was reported that viscosity influences in viscotaxis (bacteria motility) 
221 (Petrino \& Doetsch, 1978) and in microbial population composition (Wyatt \& Archer, 222 1988). \\ Conclusions}

224 Along the large intestine, there is a constant change in viscosity that is not usually taken 225 into account, for instance water is being absorbed from the small intestine till the descending colon changing viscosity in an increase fashion. These viscosity changes need to be characterized because are affecting nutrients diffusion and gut microbial development. In this work, it has been studied whether viscosity influence gut microbiota growth by supplementing the medium with bacteriological agar. It was found that depending on the species the bacteria growth was different; also bacteria changed the medium viscosity, even when keeping constant medium conditions (temperature and $\mathrm{pH}$ ). As it is influencing bacteria species growth, viscosity is a key factor to better approximate in vitro gut systems to reality. Future studies with different human microbiota donors will be needed to reinforce the hypothesis of microbiota dependence and viscosity, as well as the measure of short chain fatty acids to study the fermentation products.

\section{Acknowledgements}

The authors of this work were funded by the Spanish MINEICO project (AGL201564522-C2-R-01). L.L. would like to thank the Spanish “Juan de la Cierva” program for her contract (ref FJCI-2014-19907). C.C would like to thank the Comunidad de Madrid Program (ALIBIRD-CM S2013/ABI-278-CM) for her postdoctoral research contract. A.T. is the beneficiary of a Garantia Juvenil contract co-financed by the European Social Fund.

\section{References}

Barroso E., Cueva C., Peláez C., Martínez-Cuesta M. C., Requena T. 2015. Development of human colonic microbiota in the computer-controlled dynamic SIMulator of the GastroIntestinal tract SIMGI. LWT-Food Science and Technology, 61(2),283-289.

Benítez-Páez, A., Gómez Del Pulgar, E. M., Kjølbæk, L., Brahe, L. K., Astrup, A., Larsen, L., \& Sanz, Y. (2016). Impact of dietary fiber and fat on gut microbiota remodeling and metabolic health. Trends in Food Science \& Technology, 57, Part B, 201254212 
Beruto, M., Beruto, D., \& Debergh, P. (1999). Influence of agar on in vitro cultures: I. Physicochemical properties of agar and agar gelled media. In Vitro Cellular \& Developmental Biology - Plant, 35(1), 86. doi:10.1007/s11627-999-0016-y

Bradbeer, J. F., Hancocks, R., Spyropoulos, F., \& Norton, I. T. (2015). Low acyl gellan gum fluid gel formation and their subsequent response with acid to impact on satiety. Food Hydrocolloids, 43, 501-509.

Cueva, C., Jiménez-Girón, A., Muñoz-González, I., Esteban-Fernández, A., GilSánchez, I., Dueñas, M., Martín-álvarez, P., Pozo-Bayón, M.A., Bartolomé, B., Moreno-Arribas, M. V. (2015). Application of a new Dynamic Gastrointestinal Simulator (SIMGI) to study the impact of red wine in colonic metabolism. Food Research International, 72, 149-159.

Danielson, A. D., Newman, R. K., Newman, C. W., \& Berardinelli, J. G. (1997). Lipid levels and digesta viscosity of rats fed a high-fiber barley milling fraction. Nutrition Research, 17(3), 515-522.

Dikeman, C. L., Murphy, M. R., \& Fahey, G. C. (2006). Dietary fibers affect viscosity of solutions and simulated human gastric and small intestinal digesta. The Journal of nutrition, 136(4), 913-919.

Ellis, P., Roberts, F., Low, A., \& Morgan, L. (1995). The effect of high-molecularweight guar gum on net apparent glucose absorption and net apparent insulin and gastric inhibitory polypeptide production in the growing pig: relationship to rheological changes in jejunal digesta. British Journal of Nutrition, 74(04), 539-556.

Gallagher, M. L. (2016). Chapter 3. The Nutrients and their Metabolism. In: Krause's food \& the nutrition care process: Elsevier Health Sciences.

Hardacre, A. K., Lentle, R. G., Yap, S.-Y., \& Monro, J. A. (2016). Does viscosity or structure govern the rate at which starch granules are digested? Carbohydrate polymers, 136, 667-675.

Holloway, J. L., Lowman, A. M., \& Palmese, G. R. (2013). The role of crystallization and phase separation in the formation of physically cross-linked PVA hydrogels. Soft Matter, 9(3), 826-833.

Kim, H. T., Lee, S., Kim, K. H., \& Choi, I.-G. (2012). The complete enzymatic saccharification of agarose and its application to simultaneous saccharification and fermentation of agarose for ethanol production. Bioresource Technology, 107, 301-306.

Marinho-Soriano, E., \& Bourret, E. (2003). Effects of season on the yield and quality of agar from Gracilaria species (Gracilariaceae, Rhodophyta). Bioresource Technology, 90(3), 329-333.

Molly, K., Woestyne, M. V., Smet, I. D., \& Verstraete, W. (1994). Validation of the simulator of the human intestinal microbial ecosystem (SHIME) reactor using microorganism-associated activities. Microbial Ecology in Health and Disease, 7(4), 191-200.

Molly, K., Woestyne, M. V., \& Verstraete, W. (1993). Development of a 5-step multichamber reactor as a simulation of the human intestinal microbial ecosystem. Applied microbiology and biotechnology, 39(2), 254-258.

Petrino, M. G., \& Doetsch, R. (1978). 'Viscotaxis', a new behavioural response of Leptospira interrogans (biflexa) strain B16. Microbiology, 109(1), 113-117. 
299 Schneeman, B. O. (1987). Dietary fiber and gastrointestinal function. Nutrition 300 reviews, 45(7), 129-132.

301 Slavin, J. L. (2005). Dietary fiber and body weight. Nutrition, 21(3), 411-418.

302 Trumbo, P., Schlicker, S., Yates, A. A., \& Poos, M. (2002). Dietary reference intakes 303 for energy, carbohydrate, fiber, fat, fatty acids, cholesterol, protein and amino acids. 304 Journal of the American Dietetic Association, 102(11), 1621-1630.

305 Wyatt, G., \& Archer, D. (1988). Response of populations of human faecal bacteria to 306 viscosity in vitro. Journal of applied bacteriology, 64(2), 163-167.

307

308

309

310

311

312

313

314

315

316

317

318

319

320

321

322

323

324

325

326

327 
Table 1. Effects of agar concentration and incubation time on viscosities at 1 and $10 \mathrm{~s}^{-1}\left(\eta_{\mathrm{a}, 1}\right.$ and $\left.\eta_{\mathrm{a}, 10}\right)$ of Gut Nutrient Medium (GNM) with faecal slurries at $\mathrm{pH} 6.3$

\begin{tabular}{clll}
\hline $\begin{array}{c}\text { Agar concentration } \\
(\%, w / v)\end{array}$ & $\begin{array}{l}\text { Incubation } \\
\text { time }(\mathrm{h})\end{array}$ & $\begin{array}{l}\eta_{\mathrm{a}, 1} \\
(\text { Pa s })\end{array}$ & $\begin{array}{l}\eta_{\mathrm{a}, 10} \\
(\mathrm{~Pa} \mathrm{~s})\end{array}$ \\
\hline 0 & 0 & $0.005 \pm 0.001^{\mathrm{Db}}$ & $0.001 \pm 0.001^{\mathrm{Ca}}$ \\
0 & 24 & $0.008 \pm 0.001^{\mathrm{Ca}, \mathrm{b}}$ & $0.002 \pm 0.001^{\mathrm{Ba}}$ \\
0 & 48 & $0.009 \pm 0.001^{\mathrm{Ba}}$ & $0.002 \pm 0.001^{\mathrm{Ba}}$ \\
\hline 0.30 & 0 & $0.421 \pm 0.056^{\mathrm{Ca}}$ & $0.091 \pm 0.012^{\mathrm{Ba}}$ \\
0.30 & 24 & $0.189 \pm 0.010^{\mathrm{Bb}, \mathrm{c}}$ & $0.044 \pm 0.002^{\mathrm{Bb}}$ \\
0.30 & 48 & $0.161 \pm 0.027^{\mathrm{Bc}}$ & $0.039 \pm 0.002^{\mathrm{Bb}}$ \\
\hline 0.45 & 0 & $0.907 \pm 0.009^{\mathrm{Aa}}$ & $0.223 \pm 0.012^{\mathrm{Aa}}$ \\
0.45 & 24 & $0.572 \pm 0.051^{\mathrm{Ab}}$ & $0.144 \pm 0.012^{\mathrm{Ab}}$ \\
0.45 & 48 & $0.430 \pm 0.069^{\mathrm{Ab}}$ & $0.125 \pm 0.021^{\mathrm{Ac}}$ \\
\hline 0.60 & 0 & $0.762 \pm 0.070^{\mathrm{Bb}}$ & $0.210 \pm 0.029^{\mathrm{Ab}}$ \\
0.60 & 24 & $0.666 \pm 0.094^{\mathrm{Ab}}$ & $0.143 \pm 0.034^{\mathrm{Ab}}$ \\
0.60 & 48 & $0.548 \pm 0.114^{\mathrm{Ab}}$ & $0.143 \pm 0.029^{\mathrm{Ab}}$ \\
\hline A & 24 &
\end{tabular}

330 A-D Effect of agar concentration. For each viscosity and for the same incubation time level, mean values without the same letter are significantly different $(p<0.05)$.

a-c Effect of incubation time. For each viscosity and for the same agar concentration level, mean 


\section{Figure captions}

Figure 1. Experimental set-up showing the preparation of the Gut Nutrient Medium with different added agar concentration ([Agar] $=0 \%, 0.30 \%, 0.45 \%, 0.60 \%)$.

361 Figure 2. Flow curves of the Gut Nutrient Medium with different added agar 362 concentration $(\diamond=0 \%, \boldsymbol{\nabla}=0.30 \%, \boldsymbol{\Delta}=0.45 \%$ and $\bullet=0.60 \%$ of agar $)$ without faecal 363 slurry. Mean of the viscosity at two shear rates $\left(1\right.$ and $\left.10 \mathrm{~s}^{-1}\right)$ is also represented.

364

Figure 3. Microbial growth (in CFU/mL) in different agar concentration per day ( $0 \mathrm{~h}$ 366 marked as blue, $24 \mathrm{~h}$ marked as green and $48 \mathrm{~h}$ marked as ochre). The capital letters on 367 the different graphs signifies: A) total aerobes, B) total anaerobes, C) 368 Enterobacteriaceae, D) Enterococcus, E) lactic acid bacteria, F) Clostridium spp., E) 369 Staphylococcus aureus, H) specific faecal Lactobacillus species. 